\title{
Achilles tendon reconstruction with a half-width Achilles graft and wrap-around fascial flap
}

\author{
Theddeus Octavianus Hari Prasetyono ${ }^{1,2}$, Fransisca Sisca ${ }^{3}$ \\ ${ }^{1}$ Division of Plastic Surgery, Department of Surgery and ${ }^{2}$ Indonesian Clinical Training and Education Center (ICTEC), Cipto \\ Mangunkusumo Hospital, Faculty of Medicine Universitas Indonesia, Jakarta; ${ }^{3} \mathrm{Faculty}$ of Medicine Universitas Indonesia, Jakarta, Indonesia
}

\begin{abstract}
A 70-year-old man complained imbalance while walking, inability to perform ankle flexion, and could not stand on tip-toe 3 months after injury. The ankle looked swollen with loss of Achilles contour and obvious gait disturbance. Magnetic resonance imaging shows a $5-\mathrm{cm}$ Achilles tendon gap. Subsequently, surgery was performed to solve the neglected Achilles tendon rupture. Patient was put under general anesthesia with a regional block. Using a nontourniquet technique, a reconstructive procedure was performed using a half-width autologous Achilles tendon graft, which was attached to the calcaneal prominence with wire in a double strand Bunnell fashion. As for the proximal stump, double core Bunnell/modified Kessler suturing was carried out to suture the graft to Achilles stump. To increase the vascularization, an ipsilateral gastrocnemius fascial flap with a distally based-pedicle was harvested to wrap around the tendon graft. At a 6-month follow-up, the patient was able to stand on tip-toe and had also regained a normal gait.
\end{abstract}

Keywords Achilles tendon / Gait / Reconstructive surgical procedures / Tourniquets

\author{
Correspondence: \\ Theddeus Octavianus Hari Prasetyono \\ Division of Plastic Surgery, \\ Department of Surgery, Cipto \\ Mangunkusumo Hospital, Faculty of \\ Medicine Universitas Indonesia, J. \\ Diponegoro No 71, Jakarta 10430, \\ Indonesia \\ Tel: +62-21-31931424 \\ Fax: +62-21-31931424 \\ E-mail: teddyohprasetyono@yahoo.com
}

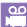

This article was presented at the First East Area Orthopedics Summit Forum on October 19-22, 2017, in Ningbo, China.

We thank Nadya Zaragita, M.D. for providing the schematic drawing.

\section{INTRODUCTION}

Patients with neglected Achilles tendon rupture have a greater tendency to experience several functional problems, specifically, gait disturbance, difficulty climbing up and down stairs, playing sports, standing on tip toes, and maintaining weight balance [13]. Thus, surgical reconstruction is strongly indicated for such a condition. Various surgical reconstructive methods are available, which mostly depend on the size of the defect and the state of the remaining tendon [3-6]. Herein, the authors report a case of a 70-year-old man with a neglected post-traumatic Achilles tendon rupture who underwent surgical treatment using an autograft taken from the Achilles tendon itself, which was wrapped with vascularized fascia.

\section{CASE}

A 70-year-old man who had remained active in his professional career experienced a gait problem after his right foot slipped into a ditch while he was running. He was unable to perform active plantar flexion, stand on tip-toe, or maintain his balance while walking. He thought that he had only sustained a sprain, so he refused any surgical intervention and had instead been undergoing traditional treatment by routine massage since the accident. He visited our clinic 3 months after the injury. On physical examination, the ankle looked swollen with a loss of the Achilles contour and obvious gait disturbance. Subsequently, magnetic resonance imaging (MRI) was performed, and showed evidence of an Achilles tendon rupture (Fig. 1). 


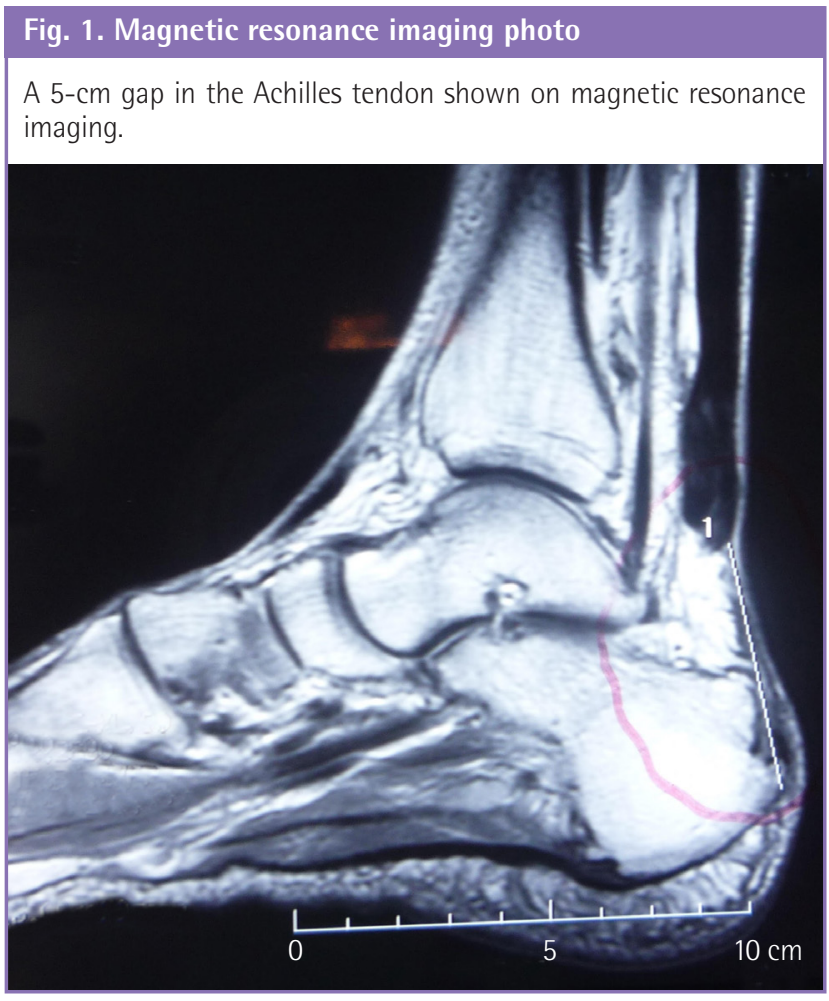

Under general anesthesia with a regional block, the patient underwent surgery in a prone position after giving consent. Using a one-per-mil tumescent injection without a tourniquet, surgery was performed in a clear operative field. The surgical approach was made through a mid-axial incision from the midpoint of the crural length to the calcaneal prominence. This revealed that the distal stump of the tendon was fragile and ragged, while the gap observed on the MRI was confirmed to be about $5 \mathrm{~cm}$ (Fig. 2A).

As there was barely any healthy Achilles tissue available at the distal stump, the author decided to take a $7-\mathrm{cm}$ half-width of Achilles tendon, to be used as a reconstructive graft (Fig. 2B). The graft was then inserted into the calcaneal prominence and secured using a 0.3-mm steel wire in a double-strand Bunnell fashion. Meanwhile, a double-core Bunnell/modified Kessler suturing technique was performed to suture the graft to the proximal Achilles stump using polypropylene 1-0. A slight bunching of the repair site was intended to ensure strong tissue contact. Subsequently, a polypropylene 4-0 running suture was used to encircle the repair site.

An ipsilateral gastrocnemius fascial flap 7-cm long and 3-cm wide with a distally-based pedicle was then harvested to wrap the tendon graft (Fig. 2C). The fascial flap was wrapped in a spiraling fashion and secured with 3-0 polyglactin 910 sutures (Fig. 2D). All of the reconstructive procedures were reassessed with a tension balance check by measuring the tension of the repaired tendon by lightly pressing the foot into dorsiflexion with the
Fig. 2. Schematic drawing of the procedure

(A) The distal stump tissue remnants were in disarray. (B) A 7-cm half-width graft was harvested to accommodate the length needed. (C) An ipsilateral gastrocnemius fascial flap was harvested. (D) The vascularized fascia was wrapped around the tendon graft in a spiraling mode.

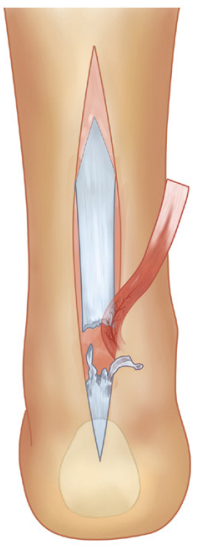

(A)

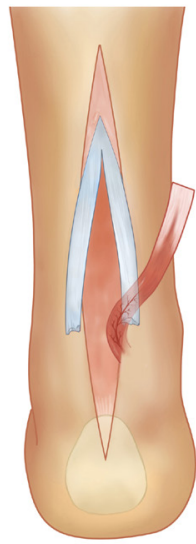

(B)
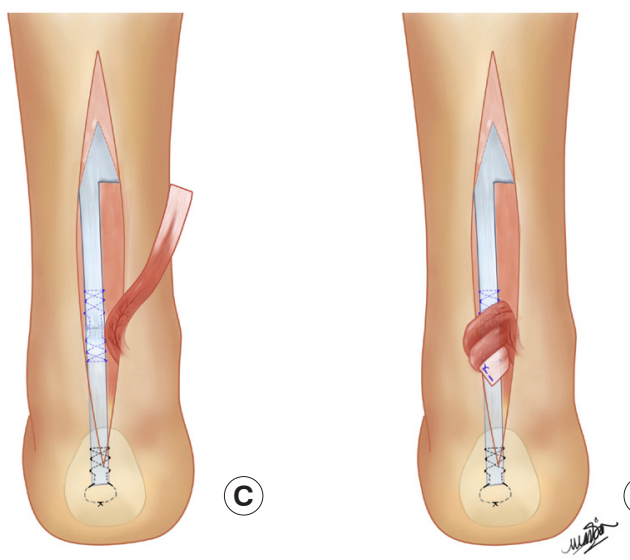

(D)

knee in $90^{\circ}$ flexion (Fig. 3). Finally, the skin was closed with a continuous intradermal suture, and the ankle was immobilized in a below-knee circular cast for 6 weeks. The surgical procedure lasted for approximately 2 hours.

During the first 6 weeks in the cast, the patient was instructed to perform isometric muscle contraction exercises. The patient wore an air boot while gradually mobilizing himself in partialweight mode during weeks 7 to 10 . Subsequently at the same time period, he was trained to perform active ankle stretching. Afterwards, the patient participated in a part-time full-weightbearing program while still wearing the air boot. In weeks 11 and 12 , the patient was in a full-time weight-bearing program. The patient did not use a cane or walker during full weight bearing.

The patient walked normally at 6 months after surgery, as shown in the attached video clip (Supplemental Video 1), and was also able to stand on tip-toe (Fig. 4). However, he still felt light pain on the anterior dorsum of the ankle when he walked 


\section{Fig. 3. Tension balance check}

An intraoperative maneuver to check the tension balance is shown.

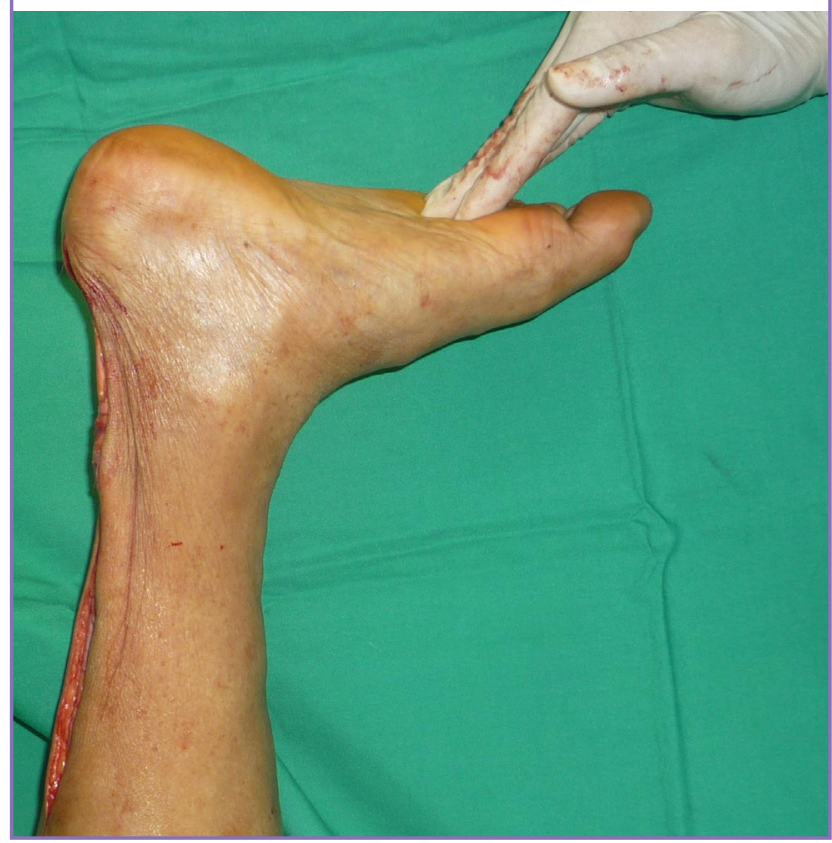

down stairs. Nonetheless, he was able to climb steps normally without any discomfort.

\section{DISCUSSION}

The goal of Achilles tendon reconstruction is to restore length, strength, and function. To elaborate, the reconstruction aims to achieve restoration of musculotendinous length and tension, to optimize soleus and gastrocnemius strength and function, and to avoid ankle stiffness [7]. Technically, surgical methods to repair a neglected Achilles tendon rupture depend on the gap size. While a gap greater than $2 \mathrm{~cm}$ might require complex surgical repair, a simple end-to-end repair could be the technique of choice when the gap between the tendon ends is less than $2 \mathrm{~cm}$. However, chronic cases with such a small tendon gap are rare, given that the tendon ends typically contract with fiber disarray. A gap between tendon ends of more than 2 to $5 \mathrm{~cm}$ most fundamentally requires myotendinous lengthening, such as the tongue-in-groove lengthening technique [3]. Surgery to reconstruct the Achilles becomes more challenging when the gap size is greater than $5 \mathrm{~cm}$. Several techniques have been reported, including tendon transfer, the use of a tendon allograft, and various other innovations. Interestingly, tendon transfer is the most widely used technique, based on the functional outcomes of donors taken from the peroneus brevis [3,8], flexor hallucis longus [8], flexor digitorum longus $[3,8]$, and tibialis anterior [8]. However, tendon transfer comes with inherent drawbacks, such

\section{Fig. 4. Standing on tip-toe}

After recovery, the patient was able to stand on his toes.

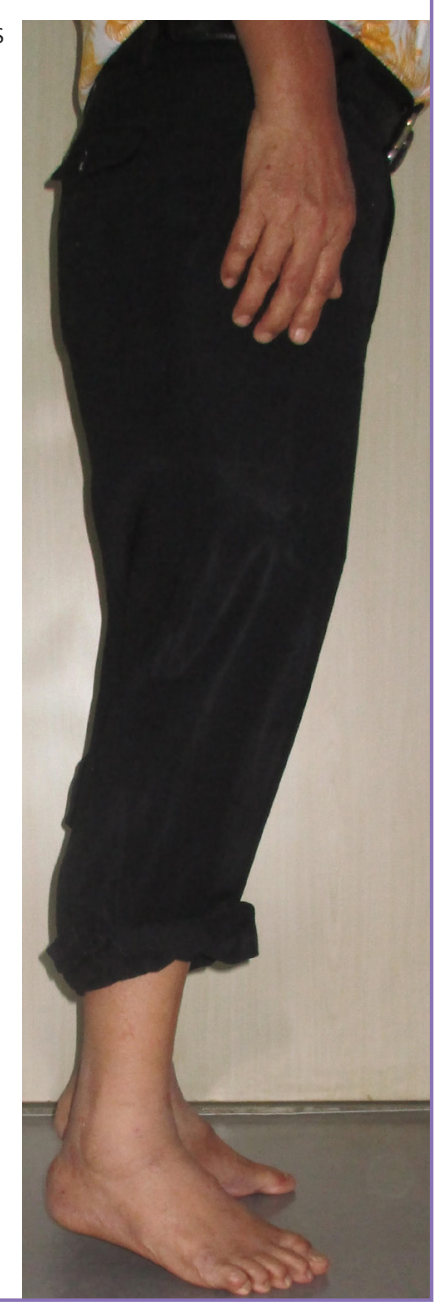

as the need to sacrifice functional structure of the donor area as well as creating a donor wound; this, in turn, weakens the motor function originally handled by the donor tendon. Most important, the reconstruction of the Achilles, which is the largest tendon in the body, will not be in balance when compared to the donor size, which is inevitably much smaller than the Achilles.

The other technique for repairing a neglected Achilles tendon rupture is an allograft. The use of an allograft has been recommended when a significant segmental defect is encountered (such as $>10 \mathrm{~cm}$ when fascia advancement or tendon transfer is unavailable to provide sufficient bridging between the tendon ends) [3]. The author did not use an allograft because of its classic susceptibility to rejection, deep infection, and the spread of infectious disease [6]. Several studies have shown that allograft tissue takes longer to incorporate; furthermore, a tendon rupture could occur if the allograft is not properly replaced by selftissue [9].

The use of synthetic materials, such as vascular grafts, carbon fiber composites, polyglycol thread, and polyester mesh, also 
have some advantages and disadvantages. The advantages are mainly not needing to sacrifice any functional tendon structure, and avoiding extensive incision and dissection [3]. However, synthetic materials are not absorbed and remodeled, potentially leading to inflammation [6].

Instead of adopting the existing techniques as reported earlier, the author used a half-width Achilles graft attached to the calcaneus, and fascial wrap-around for this case. Some justifications for this choice include a gap between tendon ends of at least 5 $\mathrm{cm}$, a lack of remaining tissue at the distal stump, and the patient's desire to avoid a donor harvest from elsewhere in the body. This technique has advantages over other methods; notably, it could be performed without needing to use the distal stump, as seen in this case, in which the tendon was in disarray. Moreover, drilling the calcaneal bone would not compromise the bone mass, as it would only require a 1-mm drill bit. On the other hand, the method requires a large volume of graft tissue, which may not be able to draw on an adequate vascular supply; thus, the vascularized fascial graft wrapped around the tendon mass is designed to support the graft take. Although postoperative MRI was not performed because of patient refusal, the final functional evaluation showed an excellent outcome.

Healing of the Achilles tendon after surgery is a complex and lengthy process. The postsurgical regimen was in accordance with a previous postsurgical rehabilitation recommendation [10], although it seemed to be less aggressive than Freedman's recommendation [11]. However, entering the 3rd month, our rehabilitation program was more aggressive than Freedman's.

At the final follow-up, the patient had achieved a normal gait, although he still felt light pain on the foot dorsum while stepping down stairs. This pain was not related to the repaired structure and might have been caused by long immobilization in a geriatric patient.

\section{NOTES}

\section{Conflict of interest}

No potential conflict of interest relevant to this article was reported.

\section{Ethical approval}

The study was performed in accordance with the principles of the Declaration of Helsinki. Written informed consent was obtained.

\section{Patient consent}

The patient provided written informed consent for the publication and the use of his images.

\section{Author contribution}

Prasetyono $\mathrm{TOH}$, study concept and design, data acquisition, study supervision, critical revision of the manuscript; Sisca F, drafting of the manuscript, critical revision of the manuscript. Approval of final manuscript: all authors.

\section{ORCID}

Theddeus Octavianus Hari Prasetyono https://orcid.org/00000003-3675-4309

Fransisca Sisca https://orcid.org/0000-0002-4366-5905

\section{Supplementary material}

Supplemental Video 1. Normal walking. The video shows the patient walking normally at follow-up.

Supplemental data can be found at: https://doi.org/10.5999/ aps.2018.00066.v001

\section{REFERENCES}

1. Pintore E, Barra V, Pintore R, et al. Peroneus brevis tendon transfer in neglected tears of the Achilles tendon. J Trauma 2001;50:71-8.

2. Tudisco C, Bisicchia S. Reconstruction of neglected traumatic Achilles tendon rupture in a young girl. J Orthop Traumatol 2012;13:163-6.

3. Lee J, Schuberth JM. Surgical treatment of the neglected Achilles tendon rupture. In: Cretnik A, editor. Achilles tendon. Rijeka: Intech; 2012. p.115-41.

4. Arriaza R, Gayoso R, Lopez-Vidriero E, et al. Quadriceps autograft to treat Achilles chronic tears: a simple surgical technique. BMC Musculoskelet Disord 2016;17:116.

5. Bevilacqua NJ. Treatment of the neglected Achilles tendon rupture. Clin Podiatr Med Surg 2012;29:291-9.

6. Park YS, Sung KS. Surgical reconstruction of chronic Achilles tendon ruptures using various methods. Orthopedics 2012;35:e213-8.

7. Mittal A. Management of TendoAchilles rupture [Internet]. c2012 [cited 2017 Oct 18]. Available from: https://www. slideshare.net/mittal87/management-of-tendoachillis-rupture.

8. Lin JL. Tendon transfers for Achilles reconstruction. Foot Ankle Clin 2009;14:729-44.

9. Mall NA, Van Thiel GS, Bedi A, et al. Graft selection in anterior cruciate reconstruction [Internet]. Rockford, IL: Ortho Illinois; c2012 [cited 2017 Oct 22]. Available from: https:// orthoillinois.com/wp-content/uploads/2012/07/GraftSelection-in-ACL-Surgery.pdf.

10. van Dijk CN. Achilles tendon repair, operative technique 
[Internet]. London: Smith \& Nephew; c2013 [cited 2017 Oct 22]. Available from: https://www.smith-nephew.com/ global/assets/pdf/products/surgical/achilles_tendon_repair_tg.pdf.

11. Freedman JA. Achilles tendon repair rehabilitation protocol
[Internet]. Rosemont, IL: American Academy of Orthopaedic Surgeons [cited 2017 Oct 22]. Available from: http:// orthodoc.aaos.org/jasonfreedmanmd/jasonfreedmanmd/ Achilles\%20tendon\%20repair.pdf. 